\title{
Euclidean preferences, option sets and strategyproofness
}

\author{
Georges Bordes • Gilbert Laffond • \\ Michel Le Breton
}

Received: 12 December 2010 / Accepted: 8 June 2011 / Published online: 23 July 2011 (C) The Author(s) 2011. This article is published with open access at SpringerLink.com

\begin{abstract}
In this note, we use the technique of option sets to sort out the implications of coalitional strategyproofness in the spatial setting. We also discuss related issues and open problems.
\end{abstract}

Keywords Social choice $\cdot$ Strategyproofness $\cdot$ Voting

JEL Classification $\quad$ D71 $\cdot$ D72 $\cdot$ D78

We are grateful to Salvador Barbera for the many stimulating scientific conversations we had over years and very pleased to offer him this little contribution which has remained unpublished. M. Le Breton would like to point out that his own interest for strategyproofness has been very much influenced by his very nice 1990 paper with Bezalel Peleg.

Our dear friend Georges Bordes passed away in 2005 . He was a very good person, a talented scholar and a friend of Salvador. He would have been very happy to offer this little present to his friend.

We would like to thank an anonymous referee for an extremely careful reading of an earlier version of this manuscript. We would also like to thank Jérome Renault and John Weymark for a very useful correspondence.

G. Laffond

Laboratoire d'Econométrie, CNAM, Paris, France

e-mail: gilbert.laffond@cnam.fr

M. Le Breton ( $ه)$

Toulouse School of Economics, Institut Universitaire de France, Toulouse, France e-mail: lebreton@cict.fr 


\section{Introduction}

The purpose of this note is to illustrate the power of an ingenious technique pioneered independently by Barbera and Peleg (1990) and Laffond (1980) and called the technique of option sets ${ }^{1}$ which has been used extensively and successfully to characterize the implications of strategyproofness in many different settings. To quote Zhou (1991), one, out of many scholars, who has used it: "It is direct and simple, invoking neither the Arrow theorem, nor any monotonicity argument. Yet, it is so powerful that under its framework many interesting issues can be addressed". Out of many contributions, ${ }^{2}$ we can cite for instance Barbera et al. $(1997,1998)$ who use the technique of option sets to investigate the case in which the domain is the class of multidimensional singlepeaked preferences and the range is a compact set and Berga and Serizawa (2000) who use it in their exploration of maximal domains for strategyproofness.

This simple notion is defined as follows. Consider a society of individuals who has to select an alternative out of a feasible set $X$ and a social choice mechanism mapping any conceivable profile $R$ of preferences into an alternative. The option set $O_{S}\left(R_{-S}\right)$ of a coalition $S$ of individuals (given the preferences $R_{-S}$ reported by the individuals outside the coalition) is the set of alternatives that they can reach through an appropriate joint report $R_{S}$ of their preferences. Therefore, the option set of coalition $S$ describes the "scope of influence" of coalition $S$ given the profile $R_{-S}$ of reports by individuals outside $S$. A social environment is characterized by a set $X$ and and a domain $\mathcal{D}$ of admissible preferences. Given a social environment $(X, \mathcal{D})$, the technique of options sets consists in sorting out gradually the properties that these different option sets must possess if the mechanism is required to satisfy some properties, on top of which some weak or strong versions of strategyproofness.

In this note, we illustrate this technique in the case where $X=\mathbb{R}^{m}$ and $\mathcal{D}$ is the set of Euclidean preferences. This result is extracted from a work that was done 20 years ago $^{3}$ (Bordes et al. 1990). The result states that, if $m \geq 2$, any surjective and coalitional strategyproof mechanism is dictatorial. The main part of the proof consists in the analysis of the case where there are two dimensions and two individuals. It uses elementary tools from basic geometry and is self contained. Most of the proof consists in showing that to prevent the mechanism to be manipulated by any one of the two individuals, say 2 , the option set of 2 must be a disk. This proof is 20 years old and, likely, some better self-contained proofs can be provided. Since then, many new results have been discovered. In the last section, we offer a brief account of some of the main achievements and open problems in this branch of the literature.

\section{The model}

We consider a society $N=\{1,2, \ldots, n\}$ of individuals who has to select an alternative in the Euclidean space $X=\mathbb{R}^{m}$. The preference $R_{i}$ of any individual $i \in N$ is entirely

\footnotetext{
1 Border and Jordan (1983) call them manipulation sets. These sets also appear in Laffont (1987).

2 I cannot cite here all the contributions who use that technique as they are too many.

3 The old version was containing a brief account of some of the results contained in the doctoral thesis of G. Laffond. In the last part of this note, we offer a short presentation of some of his ideas.
} 
described by a point $p_{i} \in \mathbb{R}^{m}$ and defined as follows: $x R_{i} y$ iff $\left\|x-p_{i}\right\| \leq\left\|y-p_{i}\right\|$ where $\|$. $\|$ denotes the usual Euclidean norm on $\mathbb{R}^{m} \cdot{ }^{4}$ The point $p_{i}$ is called the peak of individual $i$ as it is the (unique) mostly preferred alternative of individual $i$ : any individual orders alternatives according to their distance with respect to his peak. If an alternative is interpreted as the location of a public facility and $p_{i}$ as the place of residence of individual $i$, Euclidean preferences can be interpreted as preferences for smallest distances to the facility when the transportation network is unrestricted. Hereafter, we will denote by $\mathcal{E}$ the domain of Euclidean preferences over $\mathbb{R}^{m}$.

A social choice mechanism is a mapping $C$ from $\mathcal{E}^{n}$ into $X$. Since preferences in $\mathcal{E}$ are identified to their peaks, we will alternatively describe $C$ as a mapping from $\mathbb{R}^{n m}$ into $\mathbb{R}^{m}$ mapping a profile $\mathbf{p}=\left(p_{1}, p_{2}, \ldots, p_{n}\right)$ of peaks into a social alternative $C(\mathbf{p})=\left(C^{1}(\mathbf{p}), C^{2}(\mathbf{p}), \ldots, C^{m}(\mathbf{p})\right)$. Given a profile $\mathbf{p} \in \mathbb{R}^{n m}$ and a coalition $S \subseteq N$, we denote by $\mathbf{p}_{-S} \in\left(\mathbb{R}^{m}\right)^{N \backslash S}$ the restriction of the profile to $N \backslash S$. A social choice mechanism $C$ is strategyproof if there does not exist $i \in N, \mathbf{p} \in \mathbb{R}^{n m}$ and $\widehat{p}_{i} \in \mathbb{R}^{m}$ such that $\left\|C\left(\mathbf{p}_{-i}, \widehat{p}_{i}\right)-p_{i}\right\|<\left\|C(\mathbf{p})-p_{i}\right\|$. A mechanism is coalitional strategyproof if if there does not exist $S \subseteq N, \mathbf{p} \in \mathbb{R}^{n m}$ and $\widehat{\mathbf{p}}_{S} \in\left(\mathbb{R}^{m}\right)^{S}$ such that $\left\|C\left(\mathbf{p}_{-S}, \widehat{\mathbf{p}}_{S}\right)-p_{i}\right\|<\left\|C(\mathbf{p})-p_{i}\right\|$ for all $i \in S$. The range of the mechanism $C$ is the set $R(C) \equiv\left\{x \in \mathbb{R}^{m}: x=C(\mathbf{p})\right.$ for some $\left.\mathbf{p} \in \mathbb{R}^{n m}\right\}$. The mechanism $C$ is unanimous if $C(p, p, \ldots, p)=p$. $C$ is anonymous if $C\left(p_{1}, p_{2}, \ldots, p_{n}\right)=$ $C\left(p_{\sigma(1)}, p_{\sigma(2)}, \ldots, p_{\sigma(n)}\right)$ for all permutations $\sigma: N \rightarrow N$ and all $\mathbf{p} \in \mathbb{R}^{n m} . C$ is (componentwise) continuous if each component $C^{j}$ is continuous. Finally, a social choice mechanism $C$ is dictatorial if there exists $i \in N$ such that $C(\mathbf{p})=p_{i}$ for all $\mathbf{p} \in \mathbb{R}^{n m}$.

\section{The result}

Proposition If $m \geq 2$, then any coalitional strategyproof social choice mechanism $C$ over the domain $\mathcal{E}$ such that $R(C)=\mathbb{R}^{m}$ is dictatorial.

\section{The proof}

As already pointed out, the proof is based on the technique of options sets. Given a profile $\mathbf{p} \in \mathbb{R}^{n m}$ and a coalition $S \subseteq N$, we define the option set of coalition $S$ at profile $\mathbf{p}$ denoted $O_{S}(\mathbf{p})$ as the set $\left\{x \in \overline{\mathbb{R}^{m}}: x=C\left(\mathbf{p}_{-S}, \widehat{\mathbf{p}}_{S}\right)\right.$ for some $\left.\widehat{\mathbf{p}}_{S} \in\left(\mathbb{R}^{m}\right)^{S}\right\}$. It will be often denoted $O_{S}\left(\mathbf{p}_{-S}\right)$ as it only depends upon the subprofile $\mathbf{p}_{-S}$. When $S=\{i\}$ for some $i \in N, O_{S}(\mathbf{p})$ will be denoted $O_{i}\left(\mathbf{p}_{-i}\right)$. The option set of a coalition $S$, given the preferences reported by the individuals outside the coalition, is the set of alternatives attainable by the members of $S$ if they jointly control the preferences that they report. It is the range of the social choice mechanism $C_{S}\left(\mathbf{p}_{-S}\right)$ defined on $\left(\mathbb{R}^{m}\right)^{S}$. We note that $O_{N}(\mathbf{p})=R(C)$.

The following two properties are quite general (i.e. independent of the specific Euclidean setting considered here) and have been demonstrated several times. We will not repeat these arguments here.

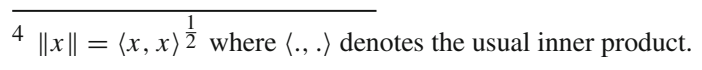


Step 1: if $p \in R(C)$, then $C(p, p, \ldots, p)=p$

Step 2 (closeness): for all $\mathbf{p} \in \mathbb{R}^{n m}$ and $S \subseteq N, O_{S}\left(\mathbf{p}_{-S}\right)$ is a closed subset of $\mathbb{R}^{m}$.

\subsection{The case where $n=m=2$}

In this section we will denote simply $O_{1}\left(p_{2}\right)$ and $O_{2}\left(p_{1}\right)$ the options set of individuals 1 and 2. Step after step, we will refine our knowledge of the options sets.

Step 3: $p_{1} \in O_{2}\left(p_{1}\right)$ and $p_{2} \in O_{1}\left(p_{2}\right)$

Step 4: $\left\|C\left(p_{1}, p_{2}\right)-p_{2}\right\| \leq\left\|p_{2}^{\prime}-p_{2}\right\|$ for all $p_{2}^{\prime} \in O_{2}\left(p_{1}\right)$ and $\| C\left(p_{1}, p_{2}\right)-$ $p_{1}\|\leq\| p_{1}^{\prime}-p_{1} \|$ for all $p_{1}^{\prime} \in O_{1}\left(p_{2}\right)$.

Step 5 (Efficiency): $C\left(p_{1}, p_{2}\right) \in\left[p_{1}, p_{2}\right]$

Step 6 (Star-shapedness): $\mathrm{O}_{2}\left(p_{1}\right)$ is star-shaped with respect to $p_{1}$.

If $O_{2}\left(p_{1}\right)=\left\{p_{1}\right\}$ the conclusion follows. Suppose that there exists $t \neq p_{1}$ such that $t \in O_{2}\left(p_{1}\right)$. We want to show that $\left[p_{1}, t\right] \subseteq O_{2}\left(p_{1}\right)$. Let $z \in\left[p_{1}, t\right]$ and assume on the contrary that $z \notin O_{2}\left(p_{1}\right)$. Since $O_{2}\left(p_{1}\right)$ is closed, so is $E \equiv[z, t] \cap O_{2}\left(p_{1}\right)$. This implies that the program Min $\|w-z\|$ has a solution, say $v$. By construction: $\left[z, v\left[\cap O_{2}\left(p_{1}\right)=\varnothing\right.\right.$. Let $\left.p_{2} \in\right] \frac{z \in E}{2}, v[$. Since $] \frac{z+v}{2}, v\left[\cap O_{2}\left(p_{1}\right)=\varnothing\right.$, we deduce from step 5 that $C\left(p_{1}, p_{2}\right) \in\left[p_{1}, z\left[\right.\right.$. Since $\left\|v-p_{2}\right\|<\left\|z-p_{2}\right\|$, this contradicts step 4 .

An immediate consequence of step 6 is the following. Given any ray $L$ with endpoint $p_{1}$, if $L \cap O_{2}\left(p_{1}\right)$ is bounded, then there exists $z=z(L)$ such that $L \cap O_{2}\left(p_{1}\right)=$ $\left[p_{1}, z\right]$.

Step 7 (Tangency): let $L$ be a ray with endpoint $p_{1}$ and $L^{\perp}(z)$ be the line orthogonal to $L$ containing $z=z(L)$. Then $O_{2}\left(p_{1}\right)$ is contained in the half plane $P(z)$ with frontier $L^{\perp}(z)$ and containing $p_{1}$, i.e. $\left\langle x-z, p_{1}-z\right\rangle \geq 0$ for all $x \in O_{2}\left(p_{1}\right)$.

Assume on the contrary that there exists $x \in O_{2}\left(p_{1}\right)$ such that:

$$
\left\langle x-z, p_{1}-z\right\rangle<0
$$

Then, there exists $y \in L$ such that $\|y-x\|<\|y-z\|$. The existence of such $y$ follows from the fact that if we take $y$ on $L$ sufficiently far from $z$, then $x$ will be in the interior of the disk centered on $y$ with radius $\|y-z\|$. This argument is illustrated in Fig. 1.

From steps 4 and 5, $C\left(p_{1}, y\right)=z$ but since $x \in O_{2}\left(p_{1}\right)$, there exists $p_{2}$ such that $x=C\left(p_{1}, p_{2}\right)$. Since $\left\|y-C\left(p_{1}, p_{2}\right)\right\|<\left\|y-C\left(p_{1}, y\right)\right\|$, this contradicts strategyproofness.

Step 8: if $O_{2}\left(p_{1}\right) \cap L$ is bounded for some $L$, then $O_{2}\left(p_{1}\right)$ is bounded.

The claim follows from a repeated application of step 7.

Step 9: if $O_{2}\left(p_{1}\right) \cap L=\left\{p_{1}\right\}$ for some $L$, then $O_{2}\left(p_{1}\right)=\left\{p_{1}\right\}$.

As for step 8, it follows from a repeated application of step 7 .

At this stage, for every $p_{1} \in \mathbb{R}^{2}$, there are three possible cases:

Case 1: $O_{2}\left(p_{1}\right)=\left\{p_{1}\right\}$

Case 2: $O_{2}\left(p_{1}\right)=\mathbb{R}^{2}$

Case 3: $O_{2}\left(p_{1}\right)$ is a compact subset of $\mathbb{R}^{2}$, star-shaped with respect to $p_{1}$ and such that $O_{2}\left(p_{1}\right) \cap L \neq\left\{p_{1}\right\}$ for all rays $L$ with endpoint $p_{1}$.

We demonstrate that case 3 cannot hold true. Suppose on the contrary that it does.

Step 10 (Convexity): $\mathrm{O}_{2}\left(p_{1}\right)$ is convex. 


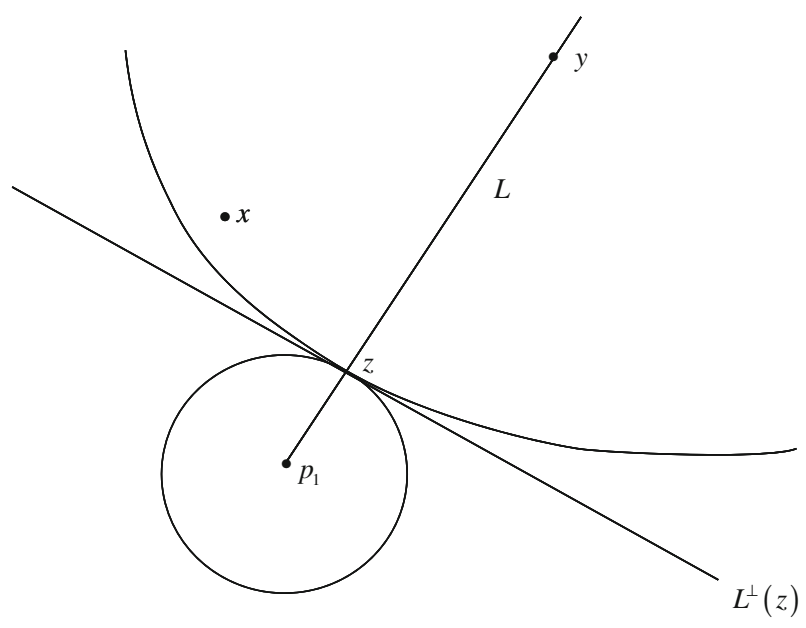

Fig. 1

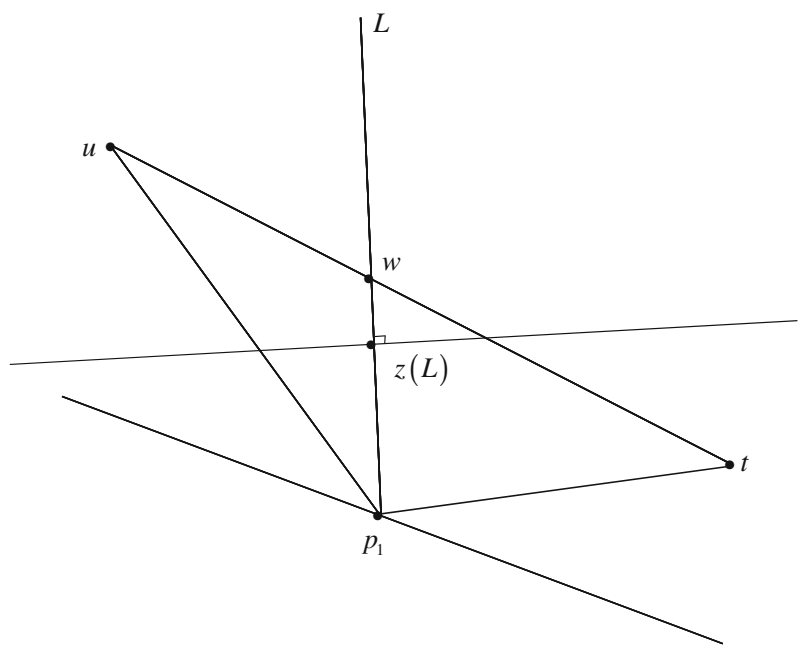

Fig. 2

Suppose on the contrary that there exist $u, t \in O_{2}\left(p_{1}\right)$ and $\left.w \in\right] t, u[$ such that $w \notin O_{2}\left(p_{1}\right)$. Consider the ray $L$ with endpoint $p_{1}$ and containing $w$. By construction, we deduce $w \notin\left[p_{1}, z(L)\right]$. In the triangle $p_{1} u t$, either $u$ or $t$ is above the line $L^{\perp}(z)$ and therefore does not belong to $O_{2}\left(p_{1}\right)$. This argument is illustrated in Fig. 2.

Let $p_{1}=(0,0)$. Let $(k, 0) \in O_{2}\left(p_{1}\right)$ be on the frontier of $O_{2}\left(p_{1}\right)$ and let $f$ : $[0, k] \rightarrow \mathbb{R}_{+}$, defined by: $f(a)=b$ where $b$ is the unique value of $c$ such that $(a, c)$ belongs to the frontier of $\mathrm{O}_{2}\left(p_{1}\right)$. From the properties of $\mathrm{O}_{2}\left(p_{1}\right)$, the function $f$ is well defined. From step 10, $f$ is concave and therefore (Rockafellar 1970), it is left differentiable and right differentiable everywhere. Let $f_{+}^{\prime}(a)$ and $f_{-}^{\prime}(a)$ be the right and left derivatives of $f$ at $a$. 


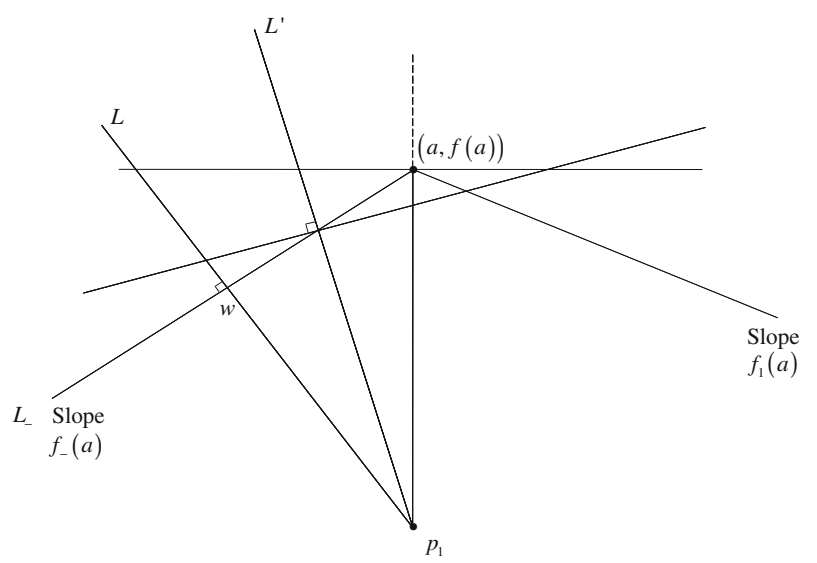

Fig. 3

Step 11: $f_{+}^{\prime}(a)=f_{-}^{\prime}(a) \equiv f^{\prime}(a)$.

Without loss of generality, assume on the contrary that $f_{+}^{\prime}(a) \leq 0<f_{-}^{\prime}(a){ }^{5}$ Let $L_{-}$be the line with slope $f_{-}^{\prime}(a)$ containing $(a, f(a))$. Since $O_{2}\left(p_{1}\right)$ is convex, it is included in the half plane with frontier $L_{-}$and containing $p_{1}$. Let $L$ be the line orthogonal to $L_{-}$containing $p_{1}$ and $w=z(L)$. Necessarily, $w=L_{-} \cap L$ as otherwise $(a, f(a)) \notin O_{2}\left(p_{1}\right)$ contradicting our assumption. From step 9, we deduce that $[w,(a, f(a))] \subseteq O_{2}\left(p_{1}\right)$. Since further $[w,(a, f(a))] \subseteq L_{-}$, we deduce that any ray $L^{\prime}$ with endpoint $p_{1}$ and intersecting $[w,(a, f(a))]$ satisfies: $z\left(L^{\prime}\right)=$ $L^{\prime} \cap[w,(a, f(a))]$. But this contradicts step 7. The argument is illustrated in Fig. 3.

From step 11, we know that $f$ is differentiable.

Step 12 (Geometry): $O_{2}\left(p_{1}\right)$ is a disk centered on $p_{1}$.

From step 7 we know that $\left(1, f^{\prime}(a)\right)$ is orthogonal to $(a, f(a))$ for all $\left.a \in\right] 0, k[$, i.e. $f$ is solution of the differential equation:

$$
a+f(a) f^{\prime}(a)=0
$$

Let $F(a)=f(a)^{2} . F$ is solution of the differential equation:

$$
2 a+F^{\prime}(a)=0
$$

whose solutions are:

$$
F(a)=C-a^{2} \text { for some constant } C
$$

and therefore:

$$
f(a)=\sqrt{C-a^{2}} \text { for some constant } C
$$

Since $f(k)=0$, we deduce that $C=k^{2}$.

\footnotetext{
$\overline{5}$ We normalize to 0 the slope of the line orthogonal to the line generated by $p_{1}$ and $(a, f(a))$ and passing through $(a, f(a))$.
} 


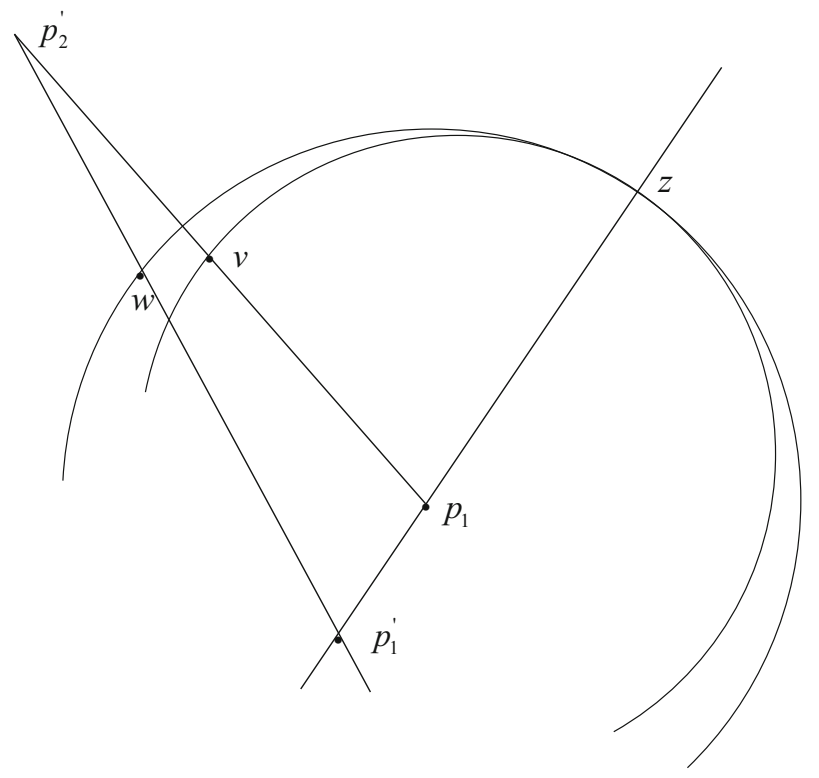

Fig. 4

Step 13: case 3 does not hold true.

From step $11 O_{2}\left(p_{1}\right)$ is a disk centered on $p_{1}$. Let $p_{2} \notin O_{2}\left(p_{1}\right), z=$ $\left[p_{1}, p_{2}\right] \cap O_{2}\left(p_{1}\right)$ and $p_{1}^{\prime} \in O_{2}\left(p_{1}\right)$ such that $p_{1} \in\left[p_{1}^{\prime}, p_{2}\right]$. From step 5, we know that $C\left(p_{1}^{\prime}, p_{2}\right) \in\left[p_{1}^{\prime}, p_{2}\right]$. Let us show that $C\left(p_{1}^{\prime}, p_{2}\right)=z$. Suppose not. Then either $C\left(p_{1}^{\prime}, p_{2}\right) \in\left[p_{1}^{\prime}, z\left[\right.\right.$ or $\left.\left.C\left(p_{1}^{\prime}, p_{2}\right) \in\right] z, p_{2}\right]$. In the first case, $\left\|C\left(p_{1}^{\prime}, p_{2}\right)-p_{1}\right\|<\left\|z-p_{1}\right\|=\left\|C\left(p_{1}, p_{2}\right)-p_{1}\right\|$ contradicting step 4. In the second case, $\left\|z-p_{1}^{\prime}\right\|=\left\|C\left(p_{1}, p_{2}\right)-p_{1}^{\prime}\right\|<\left\|C\left(p_{1}^{\prime}, p_{2}\right)-p_{1}^{\prime}\right\|$ contradicting also step 4.

Since $C\left(p_{1}^{\prime}, p_{2}\right) \notin\left\{p_{1}^{\prime}, p_{2}\right\}$, we deduce from step 11 that $O_{2}\left(p_{1}^{\prime}\right)$ is a disk centered on $p_{1}^{\prime}$. Further, since $C\left(p_{1}^{\prime}, p_{2}\right)=z$, the radius of the disk is $\left\|z-p_{1}^{\prime}\right\|$. Let $p_{2}^{\prime} \notin$ $O_{2}\left(p_{1}^{\prime}\right) \cup\left[p_{1}, p_{2}\right], v=C\left(p_{1}, p_{2}^{\prime}\right)$ and $w=C\left(p_{1}^{\prime}, p_{2}^{\prime}\right)$ as illustrated in Fig. 4.

Since $v=\left\|C\left(p_{1}, p_{2}^{\prime}\right)-p_{1}^{\prime}\right\|<\left\|C\left(p_{1}^{\prime}, p_{2}^{\prime}\right)-p_{1}^{\prime}\right\|=w$, we contradict step 4 .

At this stage, we know that for all $p_{1} \in \mathbb{R}^{2}$, either $O_{2}\left(p_{1}\right)=\left\{p_{1}\right\}$ or $O_{2}\left(p_{1}\right)=\mathbb{R}^{2}$. The following claim shows that we can invert the quantifiers.

Step 14 (Dichotomy): either $O_{2}\left(p_{1}\right)=\left\{p_{1}\right\}$ for all $p_{1} \in \mathbb{R}^{2}$ or $O_{2}\left(p_{1}\right)=\mathbb{R}^{2}$ for all $p_{1} \in \mathbb{R}^{2}$.

Assume on the contrary that there exist $p_{1}, p_{1}^{\prime} \in \mathbb{R}^{2}$ such that: $O_{2}\left(p_{1}\right)=\left\{p_{1}\right\}$ and $O_{2}\left(p_{1}^{\prime}\right)=\mathbb{R}^{2}$. Let $p_{2}$ be outside the disk centered on $p_{1}^{\prime}$ with radius $\left\|p_{1}-p_{1}^{\prime}\right\|$. We have $C\left(p_{1}^{\prime}, p_{2}\right)=p_{2}$ and $C\left(p_{1}, p_{2}\right)=p_{1}$. Since $\left\|p_{1}-p_{1}^{\prime}\right\|<\left\|p_{2}-p_{1}^{\prime}\right\|$, we contradict step 4 .

The proof of the result when $n=m=2$ is complete. When $O_{2}\left(p_{1}\right)=\left\{p_{1}\right\}$ for all $p_{1} \in \mathbb{R}^{2}, 1$ is a dictator and when $O_{2}\left(p_{1}\right)=\mathbb{R}^{2}$ for all $p_{1} \in \mathbb{R}^{2}, 2$ is a dictator. 
4.2 The case where $m=2$ and $n \geq 2$

The proof is by induction on $n$. From the preceding section, we know that the result holds true when $n=2$. We assume that it holds true when the number of individuals is less than $n-1$. Let us consider the option set $O_{-1}\left(p_{1}\right)$ of the coalition $S=N \backslash\{1\}$ when individual 1 reports the preference $p_{1}$.

Step 15: either $O_{-1}\left(p_{1}\right)=\left\{p_{1}\right\}$ or $O_{-1}\left(p_{1}\right)=\mathbb{R}^{2}$.

The proof follows with no changes the arguments from step 6 to step 13 once steps 4 and 5 have been replaced, respectively, by the following two properties:

$$
\begin{gathered}
\left\|C\left(p_{1}, p, p, \ldots, p\right)-p\right\| \leq\|x-p\| \text { for all } x \in O_{-1}\left(p_{1}\right) \\
C\left(p_{1}, p, p, \ldots, p\right) \in\left[p_{1}, p\right]
\end{gathered}
$$

The first property follows in fact from an argument similar to one implicit in step 1 applied to the mechanism $C_{-1}\left(p_{1}\right)$ while the second follows from efficiency. If $O_{-1}\left(p_{1}\right)=\left\{p_{1}\right\}$, then $O_{-1}\left(p_{1}^{\prime}\right)=\left\{p_{1}^{\prime}\right\}$ for all $p_{1}^{\prime} \in \mathbb{R}^{2}$, i.e. 1 is a dictator. Indeed, assume on the contrary that $O_{-1}\left(p_{1}^{\prime}\right)=\mathbb{R}^{2}$ for some $p_{1}^{\prime} \in \mathbb{R}^{2}$. Let $p_{2}$ be outside the disk centered on $p_{1}^{\prime}$ with radius $\left\|p_{1}-p_{1}^{\prime}\right\|$. We have $C\left(p_{1}^{\prime}, p_{2}, p_{2}, \ldots, p_{2}\right)=p_{2}$ and $C\left(p_{1}, p_{2}, p_{2}, \ldots, p_{2}\right)=p_{1}$. Since $\left\|p_{1}-p_{1}^{\prime}\right\|<\left\|p_{2}-p_{1}^{\prime}\right\|$, we contradict our assumption that $C$ is strategyproof.

If $O_{-1}\left(p_{1}\right) \neq\left\{p_{1}\right\}$, then $O_{-1}\left(p_{1}^{\prime}\right)=\mathbb{R}^{2}$ for all $p_{1}^{\prime} \in \mathbb{R}^{2}$. Since $C$ is coalitional strategyproof, $C_{-1}\left(p_{1}\right)$ is also coalitional strategyproof. Since $R\left(C_{-1}\left(p_{1}\right)\right)=$ $O_{-1}\left(p_{1}\right)=\mathbb{R}^{2}$, we deduce from the induction hypothesis that $C_{-1}\left(p_{1}\right)$ is dictatorial. Let $i\left(p_{1}\right)$ be this dictator. We now show that $i\left(p_{1}\right)$ is constant. Suppose that it is not constant and without loss of generality, assume that there exist $p_{1}, p_{1}^{\prime} \in \mathbb{R}^{2}$ such that: $i\left(p_{1}\right)=2$ and $i\left(p_{1}^{\prime}\right)=3$. Then for all $p_{2}, p_{3} \in \mathbb{R}^{2}$

$$
C\left(p_{1}, p_{2}, p_{3}, \ldots\right)=p_{2} \text { and } C\left(p_{1}^{\prime}, p_{2}, p_{3}, \ldots\right)=p_{3}
$$

If $p_{2}, p_{3}$ are such that $\left\|p_{1}-p_{3}\right\|<\left\|p_{1}-p_{2}\right\|$, then the two equalities above contradict the assumption that $C$ is strategyproof.

\subsection{The case where $m \geq 2$ and $n \geq 2$}

The proof is by induction on $m$. From the preceding section, we know that the result is true when $m=2$. We assume that it is true when the number of dimensions is less than $m-1$. Let $p \in \mathbb{R}^{n m}$. If there exists an hyperplane $H$ such that $p_{i} \in H$ for all $i=1, \ldots, n$, then we deduce from the efficiency of $C$ that $C(p) \in H$ as the convex hull of the set $\left\{p_{1}, \ldots, p_{n}\right\}$ is contained in $H$. We deduce from the induction hypothesis that the restriction $C^{H}$ of the social choice choice mechanism $C$ to $H^{n}$ is dictatorial. Let $i(H)$ be the dictator attached to $C^{H}$. We claim that $i(H)$ is constant. Take another hyperplane $H^{\prime}$. If $H^{\prime} \cap H \neq \varnothing$, then obviously $i\left(H^{\prime}\right)=i(H)$. If $H^{\prime} \cap H=\varnothing$, there exist a third hyperplane $H^{\prime \prime}$ such that $H^{\prime \prime} \cap H \neq \varnothing$ and $H^{\prime \prime} \cap H^{\prime} \neq \varnothing$. Then, $i(H)=i\left(H^{\prime \prime}\right)=i\left(H^{\prime}\right)$. Let $i(H)=1$ for all hyperplanes $H$. It remains to show that 1 is a dictator. 
If $n \leq m$, it follows from the argument above. If $n>m$, consider $p \in \mathbb{R}^{n m}$ and let $z=C(p)$. Let $z_{n} \equiv C\left(p_{1}, p_{2}, \ldots, p_{n-1}, z\right)$. We claim that $z_{n}=z$. Indeed, if $z_{n} \neq z$, then since $\left\|C\left(p_{1}, p_{2}, \ldots, p_{n-1}, p_{n}\right)-z\right\|<\left\|C\left(p_{1}, p_{2}, \ldots, p_{n-1}, z\right)-z\right\|$, we would contradict our assumption that $C$ is strategyproof. By repeating $n-m+1$ times this argument, we obtain:

$$
C\left(p_{1}, p_{2}, \ldots, p_{m-1}, z, z, \ldots, z\right)=z
$$

Since the set $\left\{p_{1}, p_{2}, \ldots, p_{m-1}, z\right\}$ is contained in an hyperplane, we conclude that $z=p_{1}$.

\section{Related literature}

In this last section, we offer a brief and selective review of the related literature and formulate some open problems.

\subsection{The case where $m=1$}

The result has been established under the assumption that there are at least two dimensions. When $m=1$, the result does not hold. There exist coalitional strategyproofness social choice mechanisms which are not dictatorial. The exploration of the class of strategyproof mechanisms in the one dimensional setting started with the seminal paper of Moulin (1980). A complete account of is large literature can be found in Barbera (2010).

\subsection{Changing the domain and/or the range}

In this paper, a social choice mechanism has been defined as a function from $\mathbb{R}^{m n}$ into $\mathbb{R}^{m}$. We could consider the general case of a function $C$ from $A^{n}$ into $B$ where $A$ and $B$ are both subsets of $\mathbb{R}^{m}: A$ and $B$ would represent, respectively, the set of admissible individual ideal points and the set of feasible social alternatives. Note that if $A=\mathbb{R}^{m}$ and $C$ is not surjective, in contrast to what has been assumed in our result, then $B$ denotes simply the range of the mechanism, i.e. $R(C)$. In Bordes et al. (1990), we prove that any coalitional strategyproof onto social choice mechanism $C: \mathbb{R}^{2} \rightarrow B$ where $B$ is an equilateral triangle is dictatorial.

To the best of our knowledge, few authors have considered the general case. The unique exception is van der Stel's thesis (1993) who offers a very general and stimulating analysis of the case where $A=B$, i.e. $C$ is a social choice mechanism from $A^{n}$ onto $A$. He proves the following generalization of our result: if $A$ is an open subset of $\mathbb{R}^{m}$, then any coalitional strategyproof onto social choice mechanism is dictatorial. His proof is different from ours and uses as auxiliary steps several other results.

The general study of the implications of coalitional strategyproofness for an arbitrary $A$ seems rather intricate. Consider the case where $A=\{(0,0),(0,1),(1,0)$, 
$\left.(1,1),\left(\frac{1}{2}, \frac{1}{2}\right)\right\}$, i.e. the set of admissible ideal points as well as the set feasible alternatives is assumed to be the set of vertices of the unit square together with its center. Consider the mechanism $C: A^{n} \rightarrow A$ defined as follows:

$$
C(\mathbf{p})= \begin{cases}p & \text { if } \mathbf{p}=(p, p, \ldots, p) \\ \left(\frac{1}{2}, \frac{1}{2}\right) & \text { otherwise }\end{cases}
$$

Since $\left(\frac{1}{2}, \frac{1}{2}\right)$ is at least second best alternative for all Euclidean preferences with an ideal point in $A$, it is easy to check that, this mechanism is coalitional strategyproof. We could object that in this example $A$ is not a product set. Having a product set does not help either. In the case where $A=\{(0,0),(0,1),(1,0),(1,1)\}$, the mechanism $C$ defined by $C(\mathbf{p})=\left(p_{1}^{1}, p_{2}^{2}\right)$ (individual 1 is a dictator on the first coordinate while individual 2 is a dictator on the second coordinate) is coalitional strategyproof. This mechanism is decomposable $e^{6}$ in the sense that the choice of the $j$ th coordinate only depends upon the vector $p^{j}=\left(p_{1}^{j}, p_{2}^{j}, \ldots, p_{n}^{j}\right)$.

The finite sets $A$ considered in the above examples are nonconvex but convexity of $A$ is not necessary for the equivalence of coalitional strategyproofness and dictatorship either, as illustrated by the the case where $A$ is the unit circle. To the best of our knowledge, the characterization of the sets $A$ for which coalitional strategyproofness is equivalent to dictatorship is still open.

\subsection{Strategyproofness and efficiency}

Can we replace, in the statement of the result, coalitional strategyproofness by (Pareto) efficiency and strategyproofness? By definition, the answer is affirmative when $n=2$. But it is not when $n \geq 3$. For instance when $X=\mathbb{R}^{2}$ and $n=3$, the social choice mechanism selecting the median of the coordinates of the ideal points for each of the two coordinates is surjective, strategyproof and Pareto efficient. Looking at our proof is quite instructive. While the coalitional strategyproofness property is preserved when we move from the mechanism $C$ to the (sub)mechanism $C_{-i}\left(p_{i}\right)$, the Pareto efficiency property is not. The induction argument which is used in our proof breaks down.

These observations raise a new question: what are the implications of the conjunction of strategyproofness and efficiency in the Euclidean setting? This question has been explored by Peters et al. (1992, 1993a,b) in a series of important papers. They consider the subclass of anonymous social choice mechanisms. One striking result that Peters et al. (1992) establishes asserts that if $C$ is a strategyproof and efficient social choice mechanism, then $C$ is continuous. Following Moulin (1980) (for the case where $m=1$ ) and Border and Jordan (1983), they define the notion of coordinatewise median scheme as follows. A collection $\left\{x^{1}, x^{2}, \ldots, x^{m}\right\} \subseteq \mathbb{R}^{m}$ is a coordinate system if $\left\langle x^{j}, x^{k}\right\rangle=0$ for all $j, k=1, \ldots, m$ with $j \neq k$, i.e. $\left\{x^{1}, x^{2}, \ldots, x^{m}\right\}$ is an orthogonal basis of $\mathbb{R}^{m}$. Let $k \in \mathbb{N}$ so that $k+n$ is odd. A social choice mechanism $C$ is a coordinatewise median social choice mechanism with $k$ constant points (phantom

\footnotetext{
6 A proof that decomposability follows from strategyproofness whenever the domain consists of a rich set of separable preferences appears in Le Breton and Sen (1999).
} 
points) if there exists a coordinate system and points $c_{1}, c_{2}, \ldots, c_{k} \in\{\mathbb{R} \cup\{-\infty, \infty\}\}^{m}$ such that:

$$
C^{j}(p)=\operatorname{Med}\left(p_{1}^{j}, p_{2}^{j}, \ldots, p_{n}^{j}, c_{1}^{j}, c_{2}^{j}, \ldots, c_{k}^{j}\right)
$$

where Med denotes the median of the subsequent real numbers and all coordinates are expressed with respect to the given coordinate system. They show that if $m \geq 2$ and $n$ is even, or if $n \geq 3$, then there does not exist surjective social choice mechanism which are anonymous, efficient and strategyproof. However when $m=2$ and $n$ is odd, the class of anonymous, efficient and strategyproof social choice mechanisms is nonempty. More precisely, the class of anonymous, efficient and strategyproof social choice mechanisms coincides exactly with the class of coordinatewise median social choice mechanism without constant points. This result was established also by Kim and Roush (1984) with the additional assumption that $C$ is continuous. But, as already pointed out by Peters, van der Stel and Storcken, this property is implied by the others and is therefore redundant.

\subsection{Strategyproofness}

Coalitional strategyproofness is often strictly more demanding than strategyproofness. It is therefore natural to wonder what is the class of strategyproof social choice mechanisms when $X=\mathbb{R}^{m}$. The coordinatewise median social choice mechanisms are strategyproof. More generally, we can construct componentwise social choice mechanisms by constructing separately a social choice mechanism for each of the $m$ components where the choice of the $j$ th social coordinate only depends upon the vector $p^{j}=\left(p_{1}^{j}, p_{2}^{j}, \ldots, p_{n}^{j}\right)$. Such mechanisms have been called decomposable as the initial problem has been decomposed into $m$ one dimensional problems. Strategyproofness follows from the fact that Euclidean preferences are separable, i.e. preferences over one coordinate do not depend upon what has been decided upon the other coordinates. Therefore, existence of nondictatorial social mechanisms is not an issue here. It is legitimate to wonder what is the exact class of strategyproof social choice mechanisms.

This a difficult question which has been addressed by Laffond (1980) in his thesis and also by Kim and Roush (1984). Kim and Roush focus on the case where $X=\mathbb{R}^{2}$. They prove that a social choice mechanism is continuous and anonymous (but not necessarily surjective) iff it is a coordinatewise median social choice mechanism with $n+1$ constant points. Peters et al. (1993b) proves that if $C$ is strategyproof then $C$ is continuous iff $R(C)$ is convex. They further point out that if $C$ is surjective then $C$ is strategyproof and anonymous iff it is a coordinatewise median social choice mechanism with $n-1$ constant points.

Laffond's work (1980) considers the case where $n=2$ but $m$ is arbitrary. $\mathrm{He}$ focuses on the class of anonymous, continuous, surjective and strategyproof social choice mechanisms. As already pointed out, to conduct his analysis, he also invented the technique of option sets. His analysis consists in a gradual exploration of the properties of the sets $O_{2}\left(p_{1}\right)$ and $O_{1}\left(p_{2}\right)$. First, he demonstrates that if $C$ is surjective, continuous and strategyproof, the option sets are closed and convex subsets of $\mathbb{R}^{m}$. 


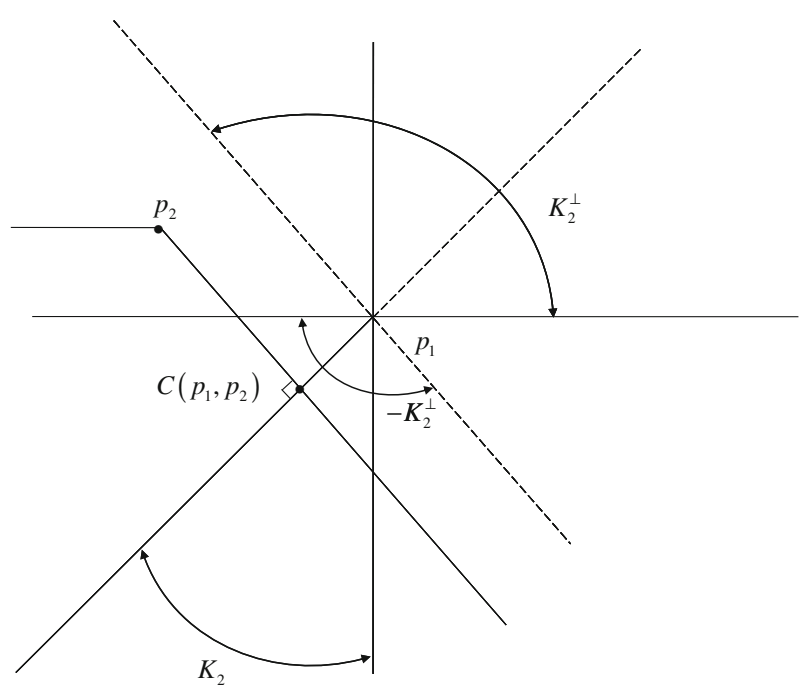

Fig. 5

This implies that $C\left(p_{1}, p_{2}\right)$ is the projection of $p_{1}$ on $O_{1}\left(p_{2}\right)$ and the projection of $p_{2}$ on $O_{2}\left(p_{1}\right)$. Of course some consistency condition is needed since the two options sets cannot be constructed independently of each other. The main part of his work consists in sorting out the implications of this consistency. We have no space here to go through all his lengthy analysis. We just sketch some of his main ideas. To show what kind of mechanisms will appear out of Laffond's exploration, consider, for the sake of illustration, ${ }^{7}$ the case where $C\left(p_{1}, p_{2}\right)$ is the projection of $p_{2}$ on $p_{1}+K_{2}$ where $K_{2}$ is the convex cone $\left\{x \in \mathbb{R}^{2}: x_{1} \leq 0, x_{2} \leq 0\right.$ and $\left.x_{2} \leq x_{1}\right\}$. By construction, $O_{2}\left(p_{1}\right)=p_{1}+K_{2}$ and $C$ is not manipulable by 2 . It is easy to show that $C$ is not manipulable either. Further, it can be verified that $O_{1}\left(p_{2}\right)=p_{2}+K_{1}$ where $K_{1}=-K_{2}^{\perp}$ where $K_{2}^{\perp} \equiv\left\{x \in \mathbb{R}^{2}:\langle x, y\rangle \leq 0\right.$ for all $\left.y \in K_{2}\right\} . K_{2}^{\perp}$ is called the polar of the cone $K_{2}$. The construction is illustrated in Fig. 5.

Another illustration is the case where individual 1 is a dictator on the first coordinate and individual 2 is a dictator on the second coordinate. In such case, $K_{2}$ is the vertical axis and $K_{1}$ is the horizontal axis. In the first example, $K_{1}$ has a nonempty interior while its is empty in the second one. These two examples violates anonymity. Anonymity prevails iff $K_{2}^{\perp}=-K_{1}$. Such cones are called self-polar (or self dual cones). Let $K$ be an arbitrary closed and convex self-polar cone with an nonempty interior. As shown by Laffond, the social choice mechanism where equivalently the social outcome is the projection of $p_{1}$ on $p_{2}+K$ or the projection of $p_{2}$ of 2 on $p_{1}+K$ is strategyproof, anonymous and continuous. This construction leads to a large family of anonymous, surjective, continuous and strategyproof social choice mechanism. What mechanisms do we find in that family?

7 This example appears in Border and Jordan (1983). 
First, and not surprisingly (from what precedes), we find the class of coordinatewise median social choice mechanism with one constant point at infinity. The self-polar cone attached to any such mechanism is (up to a rotation) the positive orthant $\mathbb{R}_{+}^{m}$. When $m=2, \mathbb{R}_{+}^{2}$ is the unique (up to a rotation) self-polar cone of $\mathbb{R}^{2}$. When $m=2$, any social choice mechanism constructed along these lines is decomposable. However, when $m \geq 3$, this is not true anymore. This follows from the fact that when $m \geq 3$, the family of self-polar cones is much richer. The analysis of these objects is a well defined area in mathematics (see, e.g. Barker and Foran 1976; Iochum 1984) and one merit of Laffond's construction is to point out this connection.

The above construction raises the following question: What is the specificity of the class of anonymous, surjective, continuous and strategyproof social choice mechanisms constructed by Laffond through self-polar cones? Laffond shows that, to answer the above question, we have to examine the asymptotic cone ${ }^{8}$ of the option set $O(p)$. In particular, he first shows that if $C$ is an anonymous, surjective, continuous and strategyproof social choice mechanism, then $O_{1}=O_{2} \equiv O$ where $O$ is a correspondence with closed and convex values such that $K(O(p))$ is independent of $p$, i.e. $K(O(p))=K\left(O\left(p^{\prime}\right)\right) \equiv K$ for all $p, p^{\prime} \in \mathbb{R}^{m}$. He further shows that if $K$ has a nonempty interior, then $C$ belongs to the class which has just been constructed, i.e. $O(p)=p+K$ and $K$ is self-polar.

\subsection{Superdomains}

The set of Euclidean preferences on $\mathbb{R}^{m}$ is in one to one correspondence with $\mathbb{R}^{m}$. Many authors have explored the questions examined in this note for sets of preferences who are supersets of the set of Euclidean preferences. One important such a superdomain is the set of diagonal (separable) quadratic preferences. They are described by utility functions $u$ such that:

$$
u(x)=-\sum_{j=1}^{m} \alpha^{j}\left(x^{j}-p^{j}\right)^{2} \quad \text { where } \alpha=\left(\alpha^{j}\right)_{1 \leq j \leq m} \in \mathbb{R}_{++}^{m}
$$

Another (larger) superdomain is the all set of quadratic preferences described by utility functions $u$ such that:

$$
\begin{aligned}
& u(x)=-\sum_{k=1}^{m} \sum_{j=1}^{m} \alpha^{k j}\left(x^{j}-p^{j}\right)^{2} \text { where } \\
& \quad \alpha=\left(\alpha^{k j}\right)_{1 \leq k, j \leq m} \text { is a symmetric positive definite matrix }
\end{aligned}
$$

Border and Jordan's (1983) seminal contribution contains many important results on these two domains. They demonstrate that a surjective social choice mechanism $C$

\footnotetext{
8 The asymptotic cone $K(A)$ of an arbitrary closed and convex subset $A$ of $\mathbb{R}^{m}$ is defined as follows: $K(A)=\left\{x \in \mathbb{R}^{m}: \forall \lambda \geq 0, \forall y \in A, x+\lambda y \in A\right\}$.
} 
over the domain of diagonal quadratic preference is strategyproof iff it is decomposable, i.e. if there exist $m$ surjective and strategyproof social choice mechanisms $C^{j}$ such that:

$$
C(\mathbf{p}, \alpha)=\left(C\left(p^{1}\right), C\left(p^{2}\right), \ldots, C\left(p^{m}\right)\right)
$$

Among other things, decomposability obliges to abandon the idea of using the information on preferences which is contained in the vectors $\alpha_{i} \in \mathbb{R}_{++}^{m}$ and therefore to limit the information on preferences to the peaks of the individual preferences. ${ }^{9}$ This decomposability result implies here a property which has been called in the literature a tops only property. ${ }^{10}$ In many other social environments settings, it is also an implication of strategyproofness.

They prove that any surjective and strategyproof social choice mechanism over the set of quadratic preferences is dictatorial. Their result has been generalized by Zhou (1991) who weakens the surjectivity assumption to a very weak range condition.

The superdomains discussed above are parametric. We could consider classes of preferences which are not described by a finite set of parameters. For example we could consider the class of preferences on $\mathbb{R}^{m}$ represented by utility functions $u$ such that:

$$
u(x)=\sum_{j=1}^{m} v^{j}\left(x^{j}\right)
$$

where each $v^{j}: \mathbb{R} \rightarrow \mathbb{R}$ has a unique maximizer from which it decreases monotonically in either direction. This domain has been explored by many authors including among others (Barbera et al. 1993). It is important to note that if it can been shown that strategyproofness implies the top only property, then we are back to the parametric domains considered earlier. Barbera (2010) offers a detailed exposition of this area of research.

Finally, we could also consider the questions explored in this note for a class of preferences generated by a distance different from the Euclidean distance. This question is explored extensively in van der Stel who considers some other norms. In location problems, the set $A$ is a assumed to be a closed and connected subset of $\mathbb{R}^{m}$ and for all $p, q \in A$, the distance between $d(p, q)$ is the shortest distance from $p$ to $q$ (and $q$ to $p$ ). Schummer and Vohra (2002) have examined the implications of surjectivity and strategyproofness in the case where $A$ is the union of a finite number of closed curves of finite length.

Open Access This article is distributed under the terms of the Creative Commons Attribution License which permits any use, distribution and reproduction in any medium, provided the original author(s) and source are credited.

\footnotetext{
9 Decomposability implies more restrictions.

10 On this, see Weymark (1999).
} 


\section{References}

Barbera S (2010) Strategyproof social choice. Handbook of social choice and welfare, vol 2, chap 25. North Holland, Amsterdam, pp 731-831

Barbera S, Gul F, Stacchetti E (1993) Generalized median voter schemes and committees. J Econ Theory 61:262-289

Barbera S, Masso J, Neme A (1997) Voting under constraints. J Econ Theory 76:298-321

Barbera S, Masso J, Serizawa S (1998) Strategyproof voting on compact ranges. Games Econ Behav 25:272-291

Barbera S, Peleg B (1990) Strategyproof voting schemes with continuous preferences. Soc Choice Welf 7:31-38

Barker GP, Foran J (1976) Self-dual cones in euclidean spaces. Linear Algebra Appl 13:147-155

Berga D, Serizawa S (2000) Maximal domains for strategyproof rules with one public good. J Econ Theory 90:39-61

Border K, Jordan J (1983) Straightforward elections, unanimity and phantom voters. Rev Econ Stud 50: $153-170$

Bordes G, Laffond G, Le Breton M, (1990) Strategyproofness issues in some economic and political domains. Mimeo, Université d'Aix-Marseille 2

Iochum B (1984) Cônes Autoplaires et Algèbres de Jordan. Lecture notes in mathematics no. 1049. Springer, Berlin

Kim KH, Roush FW (1984) Nonmanipulability in two dimensions. Math Soc Sci 8:29-43

Laffond G (1980) Révélation des Préférences et Utilit és Unimodales. Thesis, Paris

Laffont JJ (1987) Revelation of preferences. In: Eatwell J, Milgate M, Newman P (eds) The new Palgrave: a dictionary of economics, 1st edn. Palgrave MacMillan, London

Le Breton M, Sen A (1999) Separable preferences, strategyproofness and decomposability. Econometrica 67:605-628

Moulin H (1980) On strategyproofness and single peakedness. Public Choice 35:437-455

Peters H, van der Stel J, Storcken T (1992) Pareto optimality, anonymity and strategyproofness in location problems. Int J Game Theory 21:221-235

Peters H, van der Stel J, Storcken T (1993) Generalized median solutions, strategyproofness and strictly convex norms. Z Oper Res 38:19-53

Peters H, van der Stel J, Storcken T (1993) Range convexity, continuity and strategyproofness of voting schemes. Z Oper Res 38:213-229

Rockafellar RT (1970) Convex analysis. Princeton University Press, New Jersey

Schummer J, Vohra RV (2002) Strategyproof location on a network. J Econ Theory 104:405-428

van der Stel J (1993) Strategyproof voting schemes on euclidean spaces. Thesis, Maastricht

Weymark JA (1999) Decomposable strategyproof social choice functions. Jpn Econ Rev 50:343-355

Zhou L (1991) Impossibility of strategyproof mechanisms in economies with pure public goods. Rev Econ Stud 58:107-119 